\title{
Carcinoma mucoepidermoide bronquial: a propósito de dos casos clínicos
}

\author{
RAIMUNDO SANTOLAYA C.*, CAMILA SEYMOUR M.**, JAVIERA SAN MARTÍN H.***, \\ YUMAY PIRES N.****, PABLO PÉREZ C.***** y RAÚL BERRÍOS S.******
}

\section{Bronchopulmonary mucoepidermoid carcinoma: apropos of two clinical cases}

Bronchopulmonary mucoepidermoid carcinoma is an uncommon neoplasm, accounting for 0.1 to $0.2 \%$ of primary malignant tumors of the lung. In general it has a good prognosis, however there is a subtype of high grade of more ominous prognosis. In this paper we present two clinical cases of low grade pulmonary mucoepidermoid carcinoma, focused on their diagnosis and surgical management.

Key words: Carcinoma, Mucoepidermoid; Prognosis; Bronchi; Surgical management.

\section{Resumen}

El carcinoma mucoepidermoide bronquial es una neoplasia infrecuente, representando el 0,1 a $0,2 \%$ de los tumores malignos primarios del pulmón. En general tiene un buen pronóstico, sin embargo, existe un subtipo de alto grado de pronóstico más ominoso. En este artículo se presentan dos casos clínicos de carcinoma mucoepidermoide bronquial de bajo grado, enfocado en su diagnóstico y manejo quirúrgico.

Palabras clave: Carcinoma, Mucoepidermoide; Pronóstico; Bronquios; Manejo quirúrgico.

\section{Introducción}

El carcinoma mucoepidermoide pulmonar es un tumor raro, de lento crecimiento, representando solo el 0,1 al $0,2 \%$ de los tumores primarios malignos del pulmón ${ }^{1-4}$. Descrito en la literatura médica por primera vez por Smetana en $1952^{5}$, se clasifica dentro de los adenomas bronquiales ${ }^{6}$. En general es un tumor de buen pronóstico, sin embargo, existe un subtipo de alto grado que tiene un pronóstico más ominoso.

El objetivo de este artículo es presentar dos casos clínicos de pacientes con carcinoma mucoepidermoide bronquial, con enfoque en su diagnóstico y tratamiento.

\section{Casos clínicos}

\section{Caso 1}

Paciente sana, de sexo femenino, 18 años de edad. Debido a cuadros respiratorios repetidos, se decidió estudio con TAC de tórax que mostró un tumor endobronquial a nivel de bronquio lobar inferior izquierdo (Figura 1). Se realizó fibrobroncoscopía que mostró lesión endobronquial con oclusión casi completa del tronco basal izquierdo (Figura 2). La biopsia fue informada como carcinoma mucoepidermoide de bajo grado (Figuras 4 y 5). Se presentó en comité oncológico de tórax instancia donde se decidió realizar lobectomía inferior izquierda.

\footnotetext{
* Cirujano de tórax, Clínica Alemana de Santiago

** Cirujano General, Hospital FACH (Fuerza Aérea de Chile)

*** Médico Cirujano, Urgencia Hospital FACH.

**** Anátomo-patólogo. Clínica Alemana de Santiago.

***** Cirujano General. Departamento de Cirugía Occidente Universidad de Chile.

$* * * * * *$ Cirujano de tórax Clínica Alemana de Santiago y Hospital FACH.
} 


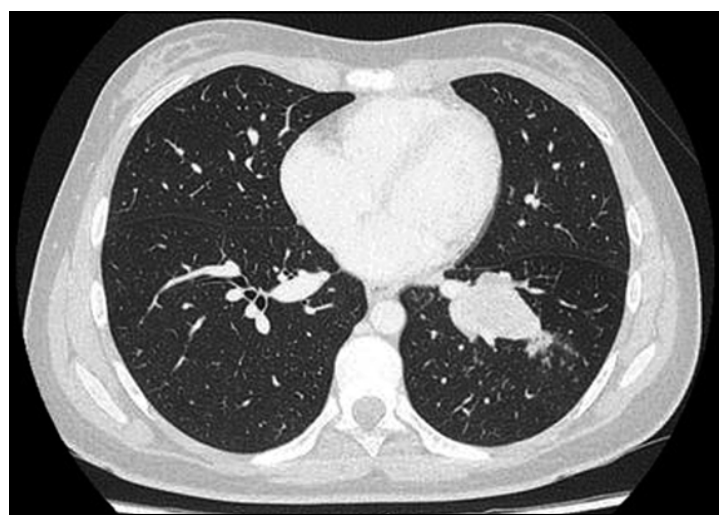

Figura 1. Tomografía computarizada (TAC) de tórax con contraste (Caso 1). Masa endobronquial de 50 x $25 \mathrm{~mm}$, que ocupa por completo y distiende el lumen del bronquio del LII, distal a la emergencia del segmentario superior. Presenta captación alta del medio de contraste y determina impactación mucosa en los bronquios hacia distal.
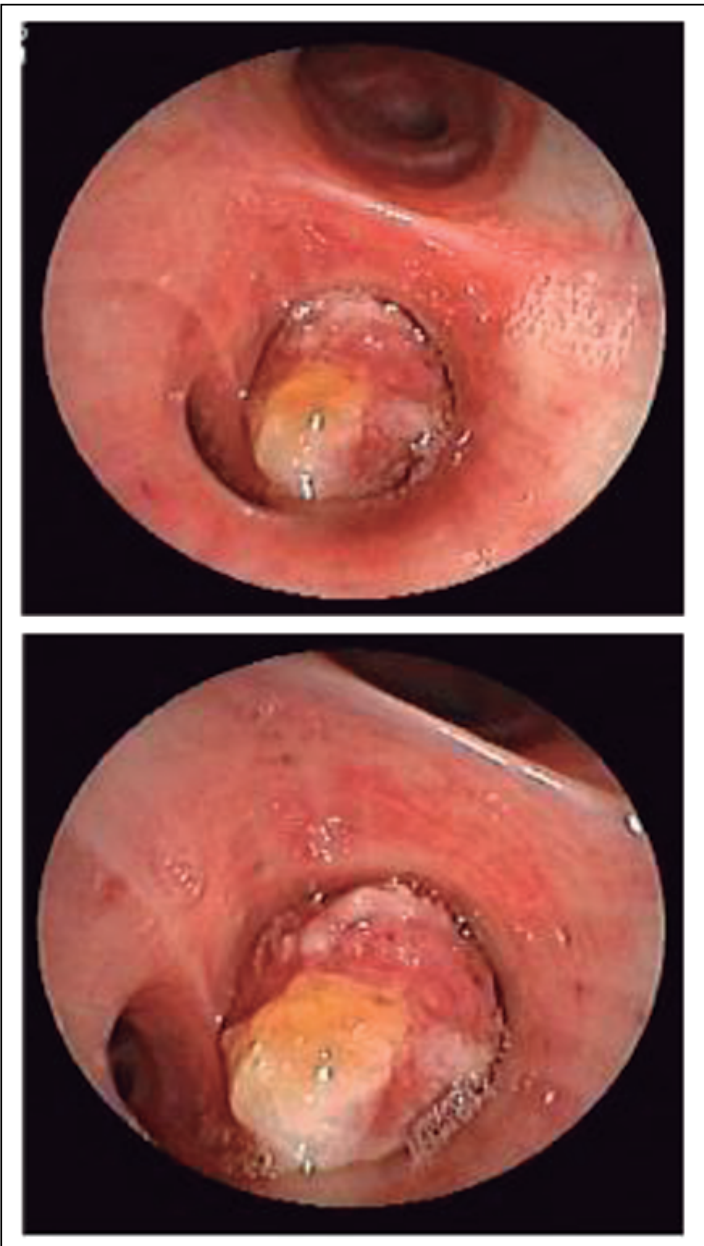

Figura 2. Fibrobroncoscopía (Caso 1). Lesión endobronquial hipervascularizada ocluyendo casi completamente el tronco de los segmentos basales del lóbulo inferior izquierdo, distal al segmento apical.
Se realizó cirugía abierta con lobectomía inferior izquierda y linfadenectomía, sin incidentes. Biopsia diferida informó carcinoma mucoepidermoide de bajo grado, de $3,2 \times 2,5 \times 1,2 \mathrm{~cm}$, sin compromiso vascular, perineural, pleural ni del borde quirúrgico. Trece ganglios linfáticos negativos (Figura 3). Se presentó en comité y decidiéndose seguimiento. Al quinto día postoperatorio se evidenció quilotórax que se manejó medicamente. Postoperatorio a un año normal.

\section{Caso 2}

Paciente sano, de sexo masculino y 20 años de edad, con historia de neumonías repetidas en lóbulo inferior izquierdo, por lo que se decidió estudiarlo. TAC de tórax mostró condensación en el segmento basal lateral y basal posterior de lóbulo inferior izquierdo en relación a lesión endobronquial en el origen de estos dos bronquios. Se completó estudio con fibrobroncoscopia y biopsia que informó carcinoma mucoepidermoide endobronquial. Se presentó en comité oncológico decidiéndose efectuar lobectomía inferior izquierda.

Se realizó cirugía abierta con la misma técnica del caso anterior. Biopsia diferida informó carcinoma mucoepidermoide endobronquial de bajo

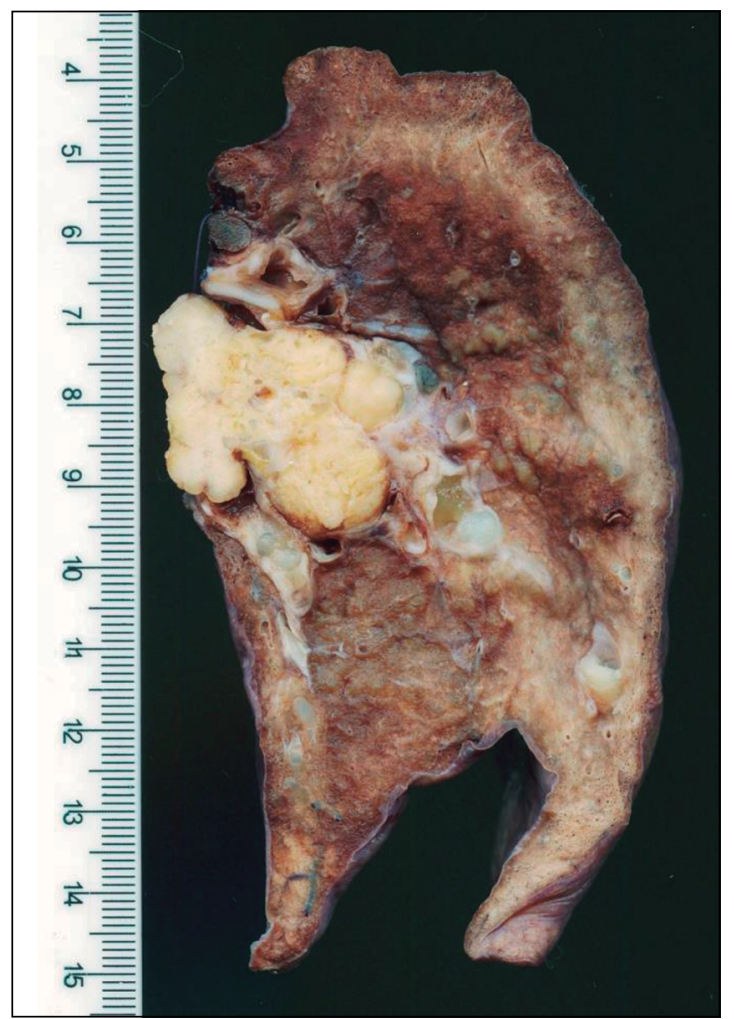

Figura 3. Pieza quirúrgica (Caso 1). Lobectomía con imagen del tumor de $3,2 \times 2,5 \times 1,2 \mathrm{~cm}$. 

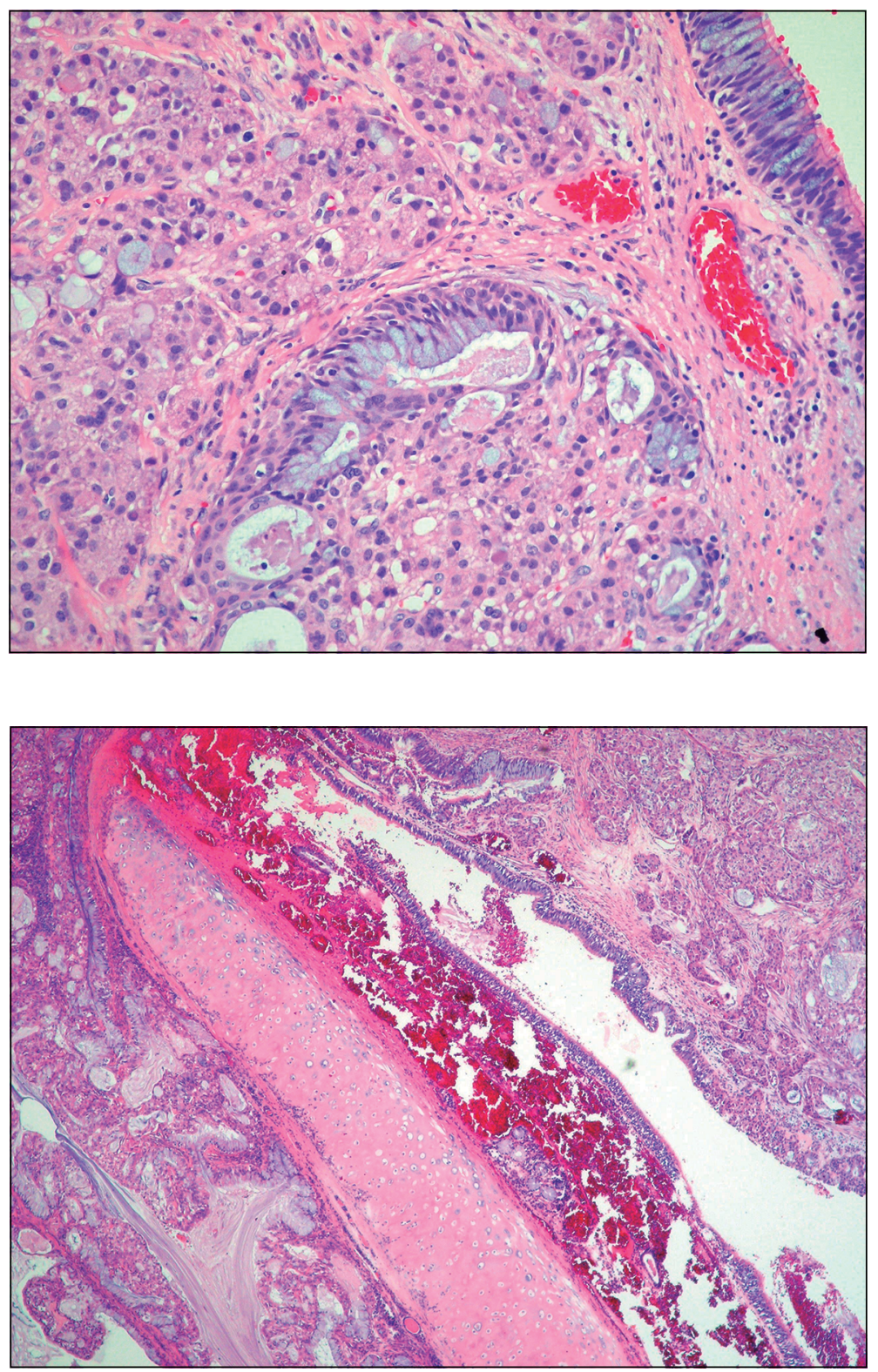

Figura 4. Microscopía con hematoxilina-eosina. Caso 1 (aumento 20x). Se observa los componentes con diferenciación mucinosa dispuestos en túbulos y en menor proporción nidos escamoides y células de tipo intermedio.

Figura 5. Microscopía con hematoxilina-eosina. Caso 1 (aumento 4x). Se observa con una visión panorámica la relación del tumor con la mucosa bronquial.

grado y presencia de siete linfonodos sin compromiso metastásico. Postoperatorio sin incidentes. Se presentó en comité oncológico, decidiéndose realizar seguimiento. Control clínico e imagenológico a 7 años fue básicamente normal.

\section{Discusión}

El carcinoma mucoepidermoide es una neoplasia de baja frecuencia en pulmón. Se origina de las glándulas mucosas y serosas del árbol traqueobronquial central, y estaría caracterizado por la combinación de células mucosecretoras, escamosas y células intermediarias ${ }^{3,7}$. No se ha logrado establecer una relación con el tabaco, ni tampoco una predilección por algún sexo ${ }^{6-8}$. Puede afectar a cualquier grupo etario, sin embargo, cerca del $50 \%$ ocurre en menores de 30 años $^{8}$, como ocurrió en los casos presentados.

Se clasifica en alto o bajo grado, según su histopatología, atipia celular, actividad mitótica, invasión local y necrosis. En general, los pacientes con carcinoma mucoepidermoide de alto grado tienen una edad mayor, y se relaciona a una mayor incidencia de metástasis linfonodales ${ }^{1}$.

Su presentación clínica está dada por síntomas derivados de la irritación y obstrucción bronquial, 
como tos, disnea, hemoptisis y neumonía ${ }^{4,6,7}$. La radiografía de tórax generalmente es anormal. Sin embargo, es esencial la TAC. Ésta muestra una masa polipoídea lobulada, no esférica, asociada a dilatación bronquial distal, impactación mucosa y atelectasia distal ${ }^{3,7}$. La biopsia se realiza a través de broncoscopía.

La resección quirúrgica es el tratamiento estándar, y últimamente el abordaje más utilizado sería por cirugía torácica video-asistida (VATS) ${ }^{1,4,9}$. El objetivo de la cirugía es la resección completa con márgenes negativos. La sobrevida global post resección es excelente con una tasa de sobrevivencia de $95 \%$ a 5 años en los carcinomas mucoepidermoides de bajo grado ${ }^{3}$.

Debido al poco número de casos reportados de carcinoma mucoepidermoide de alto grado, no existe consenso sobre terapia adyuvante especialmente para este grupo. Se propone radioterapia y/o quimioterapia adyuvante si existe invasión ganglionar o si la cirugía no ha sido curativa, sobre todo en tumores de alto grado. Sin embargo, otros afirman que la adyuvancia sería ineficaz. Por otro lado, hay algunos casos en que el inhibidor de tirosina kinasa Gefitinib ha mostrado buena respuesta en pacientes con mutaciones del gen $\mathrm{EGFR}^{4,7}$. En ambos casos presentados los tumores fueron de bajo grado, con márgenes $\mathrm{y}$ linfadenectomía negativa, por lo que se decidió solo seguimiento tras la cirugía.

Factores pronósticos relacionados a una menor sobrevivencia incluirían el grado histológico, estadio TNM, resección quirúrgica completa, $\mathrm{y}$ la edad ${ }^{1}$.

\section{Conclusiones}

El carcinoma mucoepidermoide bronquial es una neoplasia maligna rara. Debido a sus síntomas inespecíficos y su lento crecimiento, deriva en un diagnóstico difícil. Sin embargo, en general tiene un excelente pronóstico tras su resección quirúrgica aunque hay que tener en cuenta el subtipo de alto grado de pronóstico más ominoso. Se hace necesario el reporte de series más numerosas en la literatura para llegar a un consenso más acabado en el tratamiento de los carcinomas mucoepidermoides de alto grado.

\section{Bibliografía}

1.- JIANG L, LI P, XIAO Z, QIU H, ZHANG X, XIAO Y, et al. Pronostic factors of primary pulmonary mucoepidermoid carcinoma: a clinical and pathological analysis of 34 cases. Int J Clin Exp Pathol 2014; 7: 6792-9.

2.- $\quad$ LI X, ZHANG W, WU X, SUN CH, CHEN M, ZENG Q. Mucoepidermoid carcinoma of the lung: common findings and unusual appearances on CT. Clinical Imaging 2012; 36: 8-13.

3.- SHEN CH, CHE G. Clinicopathological analysis of pulmonary mucoepidermoid carcinoma. World J Surg Oncol 2014; 12: 33.

4.- KITADA M, MATSUDA Y, SATO K, HAYASHI S, ISHIBASHI K, MIYOKAWA N, et al. Mucoepidermoid carcinoma of the lung: a case report. J Cardiothoracic Surg 2011; 6: 132.

5.- SMETANA HF, IVERSON L, SWAN L. Bronchogenic carcinoma: an analysis of 100 autopsy cases. Mil Surg 1952; 111: 335-51.

6.- SÁNCHEZ I, ARCE M. Carcinoma mucoepidermoide bronquial. Diagnóstico diferencial de neumonía recurrente (Presentación de caso clínico). Rev Med Costa Rica y Centroamérica 2009; 66 (589): 261-5.

7.- CHOPRA A, SHIM CH, SHARMA N, GORDON D, TIBB A. Primary salivary type lung tumor: Mucoepidermoid carcinoma. Respiratory Medicine Case Reports. 2013; 9: 18-20.

8.- YOUSEM SA, HOCHHOLZER L. Mucoepidermoid tumors of the lung. Cancer 1987; 60: 1346-52.

9.- VADASZ P, EGERVARY M. Mucoepidermoid bronchial tumors: a review of 34 operated cases. Eur J Cardio Thorac Surg 2000; 17: 566-9.

Correspondencia a:

Dr. Raimundo Santolaya C.

Clínica Alemana de Santiago.

Avda. Vitacura 5951. Comuna de Vitacura.

Santiago, Chile.

Email: rsantolaya@alemana.cl 